This is an Open Access article distributed under the terms of the Creative Commons Attribution-Noncommercial License, which permits unrestricted use, distribution, and reproduction in any noncommercial medium, provided the original work is properly cited.

\title{
KEY SCIENCES OF SPICA MISSION: PLANETARY FORMATION, EXOPLANETS, AND OUR SOLAR SYSTEM
}

\author{
M. Tamura ${ }^{1}$, M. Takami ${ }^{2}$, K. Enya ${ }^{3}$, T. Ootsubo ${ }^{3}$, M. Fukagawa ${ }^{4}$, M. Honda ${ }^{5}$, Y. K. Okamoto ${ }^{6}$, S. \\ Sako $^{7}$, T. Yamashita ${ }^{1}$, S. Hasegawa ${ }^{3}$, H. Kataza ${ }^{3}$, Y. Doi ${ }^{8}$, H. Matsuhara ${ }^{3}$, and T. Nakagawa ${ }^{3}$ \\ ${ }^{1}$ NAOJ, Osawa 2-21-1, Mitaka, Tokyo 181-8588, Japan \\ ${ }^{2}$ ASIAA, Taipei 10617, Taiwan \\ ${ }^{3}$ ISAS/JAXA, Yoshinodai 3-1-1, Sagamihara, Kanagawa 229-8510, Japan \\ ${ }^{4}$ Oasaka University, Machikaneyama 1-1, Toyonaka, Osaka 560-0043, Japan \\ ${ }^{5}$ Kanagawa University, Tsuchiya 2946 Hiratsuka, Kanagawa 259-1293, Japan \\ ${ }^{6}$ Ibaraki University, Bunkyo 2-1-1, Mito, Ibaraki 310-8512, Japan \\ ${ }^{7}$ The University of Tokyo, Osawa 2-21-1, Mitaka, Tokyo 181-8588, Japan \\ ${ }^{8}$ The University of Tokyo, 3-8-1 Komaba, Meguro-ku, Tokyo 153-8902, Japan
}

\begin{abstract}
SPICA will provide the best sensitivity and image quality than ever at $5-210 \mu \mathrm{m}$. This will revolutionize our understanding of exoplanets, protoplanetary disks, debris disks, and Solar system small bodies. This paper summarizes such key sciences with SPICA discussed so far among the Japanese SPICA Science Working Group, stressing on the planetary formation, and exoplanet detection and characterization.
\end{abstract}

Key words: Planets: formation - Solar system: formation - infrared - Missions: SPICA

\section{INTRODUCTION}

The SPICA project stems from old activities in Japan when it was called as "H2L2 mission" around from 1999. In advance to its recent pre-Project Phase, the Science Working Group has been set up, and the "Galactic proposal" and the "Extragalactic proposal" lead by Tamura and Matsuhara, respectively, discussed the key sciences for SPICA. This was a base for the Japanese inputs to the ESA Cosmic Vision 2015-2025 SPICA Mission Proposal and the corresponding Japanese mission proposal in 2005 and 2007 (Nakagawa et al., 2008, Swinyard et al., 2009). Currently, in its Pre-Project phase, the Pre-Project team, the SPICA Task Force, the coronagraph team, and the NAOJ A-project team also discuss the key sciences rather independently. In this contribution, we will introduce some consensus on the key sciences discussed among these Japanese teams and described in the Mission Requirement Document (MRD). ISM related topics also discussed within the Galactic Proposal will be covered by Onaka's contribution in this proceedings.

\section{Research Goals and Targets}

With its large aperture and cooled telescope, the SPICA mission would provide a significant step forward in detection sensitivity in the 5 to $210 \mu \mathrm{m}$ wavelength regime, which would revolutionize our understanding of how galaxies, stars and planets form, and how interactions between complex astrophysical processes have ultimately led to the formation of our own Solar system and the emergence of life on Earth.

We now understand the basic paradigm of planet formation such as the Kyoto model or the core-accretion model, in which a heavy-element core is built by the accretion of planetesimals. As the core grows, its ability to accrete gas from the surrounding disk increases. When the core is sufficiently massive, rapid gas accretion occurs onto the core and a gas giant is formed. However, the problem with this scenario is that the time taken for gas giant formation is close to the upper limit estimated for the gas depletion timescale of the disks. In fact, planet formation is a complex process and the details are still unknown.

We therefore would like to answer the following questions, in particular. (1) What are the distributions of gas, dust, water ice and organic materials (i.e., ingredients of planets and life)? (2) How do the accumulations of dust grains and planetesimals occur? (3) What is the role of gas for the formation of planets? (4) What are the population and diversity of exoplanetary systems? (5) Are planetary systems like our Solar system common or unique?

In order to understand these questions, the key targets are exoplanets, protoplanetary disks, debris disks, and Solar System small bodies. We therefore discuss each of these in detail in the next sections. We start with a very short summary of the current understanding.

\section{Present Approaches and Problems}

\subsection{Detection And studies of exoplanets}

More than 400 exoplanet candidates have been discovered mainly using the optical radial velocity (RV) method. Indeed, 5-10 percent of the nearby main-sequence stars have giants planets (e.g., Udry et al., 2007) and the lowest masses of the discovered planets have decreased steadily down to 1.9 Earth-masses as of October 2009. However, these detections are biased in favor of exoplanets with small orbital radii and parent stars with late spectral types. 
Consequently, a number of extrasolar planetary systems might have been missed by present observational techniques and capabilities. In fact the directly imaged (outer) planets described below are difficult to be confirmed by the RV method.

The transit method provides us clues for size (thereby core density), atmospheric temperature and composition for planets (Torres et al., 2008; Swain et al., 2008) and in fact is complimentary to direct spectroscopy discussed later. Transiting spectroscopy of HD 209458b with HST revealed deep absorption in HI, OI and CII, suggesting the presence of an extended and escaping upper atmosphere beyond the Roche lobe (Vidal-Madjar et al., 2004). More recently, molecules such as $\mathrm{H}_{2} \mathrm{O}, \mathrm{CH}_{4}, \mathrm{CO}$, and $\mathrm{CO}_{2}$ have been successfully detected using the Spitzer Space Telescope and HST in transiting planet HD 189733b (Grillmair et al., 2007; Swain et al., 2008).

The transit method has recently grown significantly and about 60 exoplanets have been detected by this method to date. The number is now even dramatically increasing by the successes of Corot, Kepler, and other ground-based facilities.

Direct imaging with $8-\mathrm{m}$ telescopes and $H S T$ is also becoming successful. About 10-20 Jupiter-masses "planetbrown dwarf boundary objects" have already been imaged around 2005 (e.g., GQ Lup, DH Tau). In 2008, 3-10 Jupiter-masses "planets" around A stars (HR8799, Fomalhaut, beta Pic) were imaged and for HR8799 the three companions nearly circularly orbiting at 24, 38, $68 \mathrm{AU}$ are confirmed by others' direct imaging (Marois et al., 2008; Fukagawa et al., 2009; Close et al., 2009).

Recent ongoing and planned imaging surveys with 8$\mathrm{m}$ telescopes would even increase such directly imaged planet sample such as Gemini/NICI Planet Campaign, Subaru/HiCIAO SEEDS strategic observations (Tamura et al., 2009), and then Gemini/GPI, VLT/SPHERE. These are mostly toward nearby young ( $<100$ Myr stars); older planets are too faint and challenging at the NIR wavelengths, and those are the targets SPICA should explore.

\subsection{Protoplanetary Disks}

The studies of protoplanetary disks are changing from the SED-based one to direct imaging in these days. In fact detailed disk structures have been revealed at optical and NIR (e.g., Fukagawa et al., 2004, 2006). Its applicability is limited due to the bright stellar continuum ( $>30 \mathrm{AU}$ ), but the number of detections steady increases such as with the coronagraphs of CIAO and HiCIAO on the Subaru telescope. We will see a dramatic advance with ALMA, which will allow us to observe optically-thin thermal dust emission at AU scales. MIR imaging also works in limited cases (e.g., Fujiwara et al., 2006).

Spectroscopy of gas has been much less studied than that of dust. So far, observational studies of gas disks have been conducted mainly though radio interferometry and ground-based optical-IR spectroscopy. The former technique allows us to observe regions on a few hundred $\mathrm{AU}$ scale (e.g., Dutrey et al., 2007), while the latter allows us to observe regions within a few AU of the central star (e.g., Najita et al., 2007). Recent advances in mid-to-far IR spectroscopy have allowed us to explore the gas at intermediate radii from the stars (1-30 AU), the key zone for the formation of planetary systems like our own. Such studies include: (1) Spitzer spectroscopy of atomic ([Ne II], [Fe II] etc.) and molecular lines $\left(\mathrm{H}_{2} \mathrm{O}, \mathrm{OH}, \mathrm{HCN}, \mathrm{C}_{2} \mathrm{H}_{2}\right.$, $\mathrm{CO}_{2}$ etc.; e.g., Carr \& Najita 2008; Salyk et al., 2008); and (2) ground-based observations of [Ne II] and $\mathrm{H}_{2}$ at $17 \mu \mathrm{m}$ (Herczeg et al., 2007; Bitner et al., 2008). So, high sensitivities and spectral resolutions at MIR are highly desired to study regions with spatial scales similar to our Solar system (1-30 AU).

Spectroscopy of dust features has been pretty well explored with the 8-m telescopes and Spitzer. They have revealed silicate and $\mathrm{PAH}$ features on the disk atmosphere (Honda et al., 2006; van Boekel et al., 2008). But few cases of observations exist for water ice associated with the disks (Terada et al., 2007; Honda et al., 2009).

\subsection{DEBRIS DISKS}

Many debris disks have been discovered since the first IRAS identification of an infrared excess from the A-type main-sequence star, Vega. While most PMS stars with low-to-intermediate masses host a dust disk, a recent census by Spitzer suggests that $10-15 \%$ of nearby main sequence stars host such disks, independent of spectral type (e.g., Meyer et al., 2007). Further studies with Spitzer have marginally resolved the structure of the thermal FIR emission in the nearest and brightest debris disks (Stapelfeldt et al. 2004; $\mathrm{Su}$ et al. 2005).

There is growing evidence that such dusty disks are formed from debris produced mainly though the collisions of planetesimals in the process of planetary system formation. Since the scales of most of the observed debris disks correspond to the Kuiper belt in our Solar system, studies of these targets are important for understanding the origin and diversity of extrasolar planetary systems. More recently, the relevance of such studies has been highlighted by the discovery of a few exoplanet candidates towards stars which host bright debris disks (see Section 3.1).

While observations of debris disks are often made at far-IR and mm wavelengths, observations at shorter wavelengths (optical-MIR) have significant advantages for the study of their structure. In particular, a combination of spectroscopy and polarimetry have revealed a non-uniform distribution of dust properties, providing clues for understanding their evolution (e.g., Okamoto et al., 2004; Tamura et al., 2006). Morphological studies have been used to investigate interactions with a possible exoplanet, providing an upper mass limit (e.g., Kalas et al., 2008). However, statistical studies have been severely hampered 
by the brightness of the central star: such optical to MIR observations have been carried out for only a handful of debris disks.

See Moro-Martin's contribution in this proceedings for more details.

\subsection{Solar SYSTEM SMALL BODIES}

Since the first discovery of Trans-Neptunian Objects (TNO) in the outer Solar system (Jewitt \& Luu, 1993), more than a thousand such objects have been detected, including some of planetary size (e.g., Bertoldi et al., 2006; Brown et al., 2006). However, physical properties for most of the TNOs are not well known. Thermal imaging and photometry would allow us to determine the sizes and albedos of TNOs. With AKARI only less than 15 objects and with Spitzer less than 50 objects have been studied and detections at several bands are much less.

\section{Toward Thorough Understanding with SPICA}

\subsection{Objective No.1: Exoplanets}

To understand the diversity of the planetary systems, we will attempt to directly detect/characterize exoplanets and to measure their atmospheric composition in the infrared wavelengths.

With the planet/star contrast ratio of $10^{-6}$ or better, that will be realized with the SPICA MIR coronagraph (Tamura et al., 2000; SCI, Enya et al., 2008), we will directly detect gas exoplanets, and perform their spectroscopic observations to clarify the composition of the atmosphere such as $\mathrm{CH}_{4}$ at $7.7 \mu \mathrm{m}, \mathrm{H}_{2} \mathrm{O}$ at $6.3 \mu \mathrm{m}$, and $\mathrm{NH}_{3}$ at $6.1,10.3,10.6 \mu \mathrm{m}$. Considering the rather limited spatial resolutions restricted by the telescope aperture smaller than JWST and 8-m ground-based telescopes (or coming 30-m telescopes) and the telescope optimized for FIR and MIR, the best targets for the SPICA coronagraph must be relatively older (up to $\sim 1$ Gyr) most nearby stars. In particular, its high contrast spectroscopic capability is unique.

Comparison with the results on our Solar system planets as well as the known exoplanets enables us to reveal the diversity of the planetary systems.

With the spectroscopic observations utilizing the transit method, we will try to detect the atmosphere of giant Earth-like planets. We will also apply the same approach to gas giant planets for detailed studies of their atmosphere. The instruments available for the transit studies are MIRACLE grism, MIRMES, FPC-S grism, MIRHES, and SCI non-coronagraph spectroscopy.

Direct 5-20 $\mu \mathrm{m}$ spectroscopy of $<10$ Jupiter masses exoplanets up to 5 Gyr must be unique in SPICA. This is complimentary to JWST, TMT or $8 \mathrm{~m}$ telescopes with extreme adaptive optics (ExAO). SPICA will certainly fill the wavelength gap between Herschel and JWST and plays important and unique role after the life time of JWST for this kind of science (with both direct and indirect approaches), too.

\subsection{Objective No.2: Gas in Disks}

We will reveal the formation mechanism of gas giant planets and initial condition of terrestrial planet formation, by observing the dissipation of gas and their structural evolution in planet-forming.

With unprecedented sensitivity at $20-40 \mu \mathrm{m}$, we will survey for emission lines which could be (or are) associated with warm gas (100-1000 K) in protoplanetary disks. Using lines associated with disks, we will measure the amount of gas and how it varies with stellar mass and age. We will elucidate the geometric, physical and chemical structure of proto-planetary disks by measuring the motion of gas with high-dispersion infrared spectroscopy.

Theories suggest important roles of gas for planet formation. These include (1) gravitational instability which could form planets without dust accumulation, and (2) gas drag on solid material, determining the population and orbits of terrestrial planets.

IR Spectroscopy at $4-40 \mu \mathrm{m}(\mathrm{R} \sim 1,000)$ with MIRMES is a powerful tool for observing planet-forming regions (12-160 $\mu \mathrm{m}$ maximum; 24-63 $\mu \mathrm{m}$ minimum; $10^{-19} \mathrm{~W}$ $\mathrm{m}^{-2}$ ). Many probes exist such as $\mathrm{H}_{2} \mathrm{O}, \mathrm{CO}$, [NeII], $\mathrm{H}_{2}$, $\mathrm{C}_{2} \mathrm{H}_{2}, \mathrm{HCN}, \mathrm{CO}_{2}, \mathrm{H}_{2} \mathrm{O}$, and ionic emission (Carr and Najita, 2008).

The superb detection limits of the SPICA spectrographs are sufficient for observing MIR-FIR lines predicted by disk models, even for those whose IR excess is diminishing. High-resolution (R 30,000) spectroscopy with MIRHES would allow us to observe the evolution of disk structure due to planet formation.

\section{3. Овjective No.3: Dust in Disks}

We reveal the similarity or diversity of extrasolar systems by observing a number of debris disks, which are much more easily observable than exoplanets.

With the help of 3 times or more higher spatial resolution and 10 times or more higher sensitivity than AKARI, we will detect a number of disks whose amount of dust is even comparable to our Solar system, leading us to understand the relationship with planetary systems observed using the other methods.

Faint debris disks around normal stars can be detected by imaging observations ( 230 objects, $10-100 \mu \mathrm{m})$. Zodiacal light equivalents may not be detected. due to the limited spatial resolution but Kuiper Belt debris should be detected with SAFARI. They provide us clues on the current status of planet formation. Current disposition of planets may also be inferred.

SAFARI FIR imaging and SCI MIR coronagraphic imaging would allow the study of the geometrical and physical 
structure of bright debris disks in detail. The morphological information provided by such an instrument would be used to study the origin of the diversity of debris disks, and determine the presence or absence of large gas-giant planets near such disks. The field of view of these instruments $\left(2^{\prime} \times 2^{\prime}\right.$ for SAFARI, FIR; $1^{\prime} \times 1^{\prime}$ for coronagraph, MIR) is sufficient to cover such disks in a single frame. The sensitivity of SPICA at FIR will be higher than AKARI and Spitzer by a factor of 10, thus it will dramatically increase the sample of known debris disks.

\subsection{OBjective No.4: ICE IN Disks}

We will reveal the role of ice for planet formation, and how the elements for originating and sustaining life could be supplied to terrestrial protoplanets.

We will apply high-contrast IR coronagraphy to protoplanetary disks and debris disks, observe their structures, and understand their relationship for disk evolution. Through infrared spectroscopic observations with 3 times or higher spatial resolution than AKARI, we will reveal distribution and physical state of solid materials, particularly ice, in proto-planetary disks and dust disks in the main-sequence stars.

SAFARI Spectro-imaging of the $44 / 62 \mu \mathrm{m}$ ice features towards the brightest debris disks could be used to infer the presence or absence of the "snow-line", the possible boundary between terrestrial and gas-giant planets.

SAFARI could also be used to observe ice features at $44 / 62 \mu \mathrm{m}$ for a number of protoplanetary disks. These features hold the key to identifying the nature of the ice (either amorphous or crystalline), and are therefore useful for determining the thermal (and thereby chemical) history of the disks.

\subsection{Objective No.5: Solar System Small Objects}

In order to reveal the whole picture of the Solar system, we will survey physical information for primordial objects in the Solar system.

With the help of 10 times or more higher sensitivity than AKARI, we will make an unprecedented survey of albedo, size, thermal inertia, and surface composition for primitive objects in the Solar system.

The diameter and albedo of these objects will allow us to investigate the initial conditions and dynamical evolution of the Solar system. In order to determine the size, albedo and thermal inertia of unresolved small bodies, both the visible and thermal infrared brightness have to be measured. In particular, the measurement of the SED, which peaks at $\sim 100 \mu \mathrm{m}$, dramatically decreases the uncertainties in the determination of the size and albedo. These observations require imaging or photometric facilities at far-IR $(30-300 \mu \mathrm{m})$ wavelengths. Spitzer had only a few photometric bands in this range $(24 / 70 / 160 \mu \mathrm{m})$, while Herschel will not be able to observe wavelengths shorter than $60 \mu \mathrm{m}$.

SPICA/SAFARI would be ideally suited to the observations of the SEDs of TNOs with unprecedentedly high sensitivity and accuracy. Such observations of a large number of TNOs would probe the conditions of the 'Initial Solar Nebula' in much greater detail than previously accomplished. Furthermore, low-resolution spectroscopy of the $44 / 62 \mu \mathrm{m}$ water ice features would facilitate the study of the water ice content and thermal history of the outer Solar system.

\section{ACKNOWLEDGEMENTS}

We thank Y. Aikawa, A. Kouchi, H. Izumiura, T. Miyata, Y. Okada, T. Onaka, I. Sakon, N. Kobayashi, J. Watanabe, T. Kotani, N. Narita, T. Matsuo, Y. Itoh, J. Nishikawa, N. Murakami for various discussions and instrument advices. MT was supported by MEXT, Grant-in-Aid for Scientific Research on Priority Areas. SH was supported by Space Plasma Laboratory, JAXA/ISAS.

\section{REFERENCES}

Bertoldi, F., Altenhoff, W., Weiss, A. 2006, Nature, 439, 563

Bitner, M. A., Richter, M. J., Lacy, J. H. 2008, ApJ, 688, 1326

Brown, M. E., Schaller, E. L., Roe, H. G. et al. 2006, ApJ, 643, L61

Bryden, G., Chen, X., Lin, D. N. C. et al. 1999, ApJ, 514, 334

Carr, J. S., Najita, J. R. 2008, Science, 319, 1504

Close, L. et al. 2009, astro-ph 0904.3936

Dutrey, A., Guilloteau, S., Ho, P. 2007, Protostars and Planets V, p.495-506

Enya, K., Abe, L., Haze, K. et al. 2008, Proceedings of the SPIE, Volume 7010, pp. 70102Z-70102Z-10

Fujiwara, H., Honda, M., Kataza, H. et al. 2006, ApJ, 644, L133

Fukagawa, M., Hayashi, M., Tamura, M. et al. 2004, ApJ, 605, L53

Fukagawa, M., Tamura, M., Hayashi, M. et al. 2006, ApJ, 636, L153

Fukagawa, M., Itoh, Y., Tamura, M. et al. 2009, ApJ, 696, L1

Grillmair, C. J., Charbonneau, D., Burrows, A. et al. 2007, ApJ, 658, L115

Herczeg, G. J., Najita, J. R., Hillenbrand, L. A. 2007, ApJ, 670, 509

Honda, M., Kataza, H., Okamoto, Y. K. et al. 2006, ApJ, 646, 1024

Honda, M., Inoue, A. K., Fukagawa, M. et al. 2009, ApJ, 690, L110

Jewitt, D., Luu, J. 1993, Nature, 362, 730

Kalas, P., Graham, J. R., Chiang, E. et al. 2008, Science, 322, 1345 
Marois, C., Macintosh, B., Barman, T. et al. 2008, Science, 322,1348

Meyer, M. R., Backman, D. E., Weinberger, A. J. 2007, Protostars and Planets V, p.573-588

Najita, J. R., Carr, J. S., Glassgold, A. E. et al. 2007, Protostars and Planets V, p.507-522, 2007.

Nakagawa, T. 2008, SPICA mission for mid- and farinfrared astronomy, SPIE, 7101, 70100H-70100H-8

Okamoto, Y., Kataza, H., Honda, M. et al. 2004, Nature, 431, 660

Salyk, C., Pontoppidan, K. M., Blake, G. A. et al. 2008, ApJ, 676, L49

Su, K. Y. L., Rieke, G. H., Misselt, K. A. et al. 2005, ApJ, 628,487

Stapelfeldt, K. R., Holmes, E. K., Chen, C. et al. 2004, ApJS, 154, 458

Swain, M. R., Vasisht, G., Tinetti, G. 2008, Nature, 452 , 329

Swinyard, B., et al. 2009, Experimental Astronomy, 23, 193

Tamura, M. 2000, ISAS Report, SP, No. 14, pp 1-9

Tamura, M. 2009, Exoplanets and Disks: Their formation and Diversity, AIP Conference Proceedings, 1158, pp $11-16$

Tamura, M., Fukagawa, M., Kimura, H. et al. 2006, ApJ, 641, 1172

Terada, H., Tokunaga, A. T., Kobayashi, N. et al. 2007, ApJ, 667, 303

Torres, G. Winn, J. N., Holman, M. J. 2008, ApJ, 677, 1324

Vidal-Madjar, A., Desèrt, J. -M., Lecavelier des Etangs, A. et al. 2004, ApJ, 604, L69

van Boekel, R., Min, M., Waters, L. B. F. M. et al. 2005, A\&A, 437, 189

Udry, S., Fischer, D., Queloz, D. 2007, Protostars and Planets V, p.685-699 